\title{
Fatores contribuintes e riscos associados em caso de tomada de decisão por enfermeira durante o transporte inter-hospitalar de paciente acometido por COVID-19
}

Contributing factors and associated risks in case of decision-making by a nurse during the interhospital transport of a patient affected by COVID-19

Factores contribuyentes y riesgos asociados en caso de toma de decisiones por parte de una enfermera durante el traslado interhospitalario de un paciente afectado por COVID-19

\section{Resumo}

A complexidade das ações no cenário do atendimento inter-hospitalar impõe uma série de questões éticas às enfermeiras, sendo estas relacionadas à assistência aos clientes, ao ambiente de trabalho e aos processos organizacionais. Adicionalmente, o atual estado de emergência em saúde, caracterizado pela pandemia de COVID-19 determina reorganizações no processo de trabalho e aumento da tensão emocional das profissionais. O objetivo deste estudo foi analisar os fatores contribuintes e os riscos associados no caso de tomada de decisão por uma enfermeira durante transporte inter-hospitalar de paciente acometido por COVID-19, divulgado nos meios de comunicação brasileiros. Estudo descritivo, teórico e reflexivo, a partir da análise de vídeos e matérias jornalísticas, baseado no diagrama de causa e efeito (diagrama de Ishikawa) e na matriz de avaliação de riscos, desenvolvido entre os meses de fevereiro e março de 2021. Foram identificadas as causas para a tomada de decisão da profissional diante de uma situação crítica no atendimento inter-hospitalar, sendo a principal delas a interdição da estrada. A análise dos riscos aos quais a enfermeira e o paciente ficaram expostos permitiu reflexão sobre a importância de um suporte adequado aos profissionais durante o atendimento inter-hospitalar e de um planejamento minucioso para esta situação, de maneira a minimizá-los e garantir a segurança para todos os envolvidos.

Palavras-chave: Cuidados de enfermagem; Transferência de clientes entre hospitais; Segurança do paciente; Tomada de decisão clínica.

\footnotetext{
Abstract

The complexity involved in inter-hospital care imposes a series of ethical issues on nurses, which are related to customer care, the work environment and organizational processes. Additionally, the current state of health emergency, characterized by the COVID-19 pandemic, determines reorganizations in the work process and an increase in the emotional tension of professionals. The aim of this study was to analyze the contributing factors and
} 
associated risks in the case of decision-making by a nurse during inter-hospital transport of a patient affected by COVID-19, published in the Brazilian media. Descriptive, theoretical and reflective study, supported on videos and journalistic articles, based on the cause and effect diagram (Ishikawa diagram) and on the risk assessment matrix, developed between February and March 2021. The causes of the decision-making of the professional in the face of a critical situation in inter-hospital care were identified., the main one being the interdiction of the road. The analysis of the risks to which the nurse and the patient were exposed allowed reflection on the importance of adequate support for professionals during inter-hospital care and a detailed planning for this situation, in order to minimize them and ensure safety for everyone involved.

Keywords: Nursing care; Patient transfer; Patient safety; Clinical decision-making.

\section{Resumen}

La complejidad de las acciones en el contexto de la atención interhospitalaria impone una serie de cuestiones éticas al enfermero, que se relacionan con la atención al cliente, el entorno laboral y los procesos organizativos. Adicionalmente, el actual estado de emergencia sanitaria, caracterizado por la pandemia COVID-19, condiciona reorganizaciones en el proceso de trabajo y un aumento de la tensión emocional de los profesionales. El objetivo de este estudio fue analizar los factores contribuyentes y riesgos asociados en el caso de la toma de decisiones por parte de una enfermera durante el traslado interhospitalario de un paciente afectado por COVID-19, publicado en los medios brasileños. Estudio descriptivo, teórico y reflexivo, soportado en videos y artículos periodísticos, basado en el diagrama de causa y efecto (diagrama de Ishikawa) y en la matriz de evaluación de riesgos, desarrollado entre febrero y marzo de 2021. Las causas para la toma de decisiones del profesional frente a una situación crítica en la atención interhospitalaria fueron identificadas, siendo la principal la interdicción de la vía. El análisis de los riesgos a los que estuvieron expuestos la enfermera y el paciente permitió reflexionar sobre la importancia de un adecuado acompañamiento a los profesionales durante la atención interhospitalaria y una planificación detallada de esta situación, con el fin de minimizarlos y garantizar la seguridad de todos los involucrados.

Palabras clave: Atención de enfermería; Transferencia de pacientes; Seguridad del paciente; Toma de decisiones clínicas.

\section{Introdução}

No Brasil, a rede de atenção à saúde (RAS) compreende uma organização de serviços de saúde e atividades com a finalidade de garantir a integralidade do cuidado, baseando-se em uma relação horizontal entre os pontos da rede (Brasil, Ministério da Saúde, 2010). Um destes pontos é a rede de atenção às urgências (RAUE), que caracteriza o atendimento préhospitalar como uma ferramenta fundamental para reduzir possíveis sequelas e garantir a sobrevida de pacientes que possuem agravos à saúde (Brasil, Ministério da Saúde, 2011; Souza, Teles \& Oliveira, 2020).

Um fator importante para o bom funcionamento das RAS é a regulação em saúde, que estabelece uma articulação entre a gestão e a assistência. A regulação em saúde viabiliza a conformação de diferentes arranjos assistenciais, adaptados de acordo com as características de cada região buscando cumprir os princípios da eficiência e efetividade do sistema. Ou seja, a qualidade da assistência, a segurança dos pacientes e o bom funcionamento das RAS dependem fortemente do processo de regulação em saúde (Macedo et al, 2020).

A Política Nacional de Regulação (PNR) está fundamentada em três dimensões: regulação da atenção à saúde, regulação de sistemas de saúde e regulação do acesso à assistência, que possuem a função de apoiar a organização do sistema de saúde, otimizar recursos e qualificar a atenção e o acesso da população aos serviços. A regulação da assistência permite um melhor controle do sistema e com isso organizar e estruturar as unidades do menor para o maior nível de complexidade, respeitando os princípios da eficiência, eficácia e efetividade (Brasil, Ministério da Saúde, 2008; Barbosa, Barbosa \& Najberg, 2016).

Neste sentido, com o início da Pandemia de COVID-19, a partir de fevereiro de 2020, municípios e regiões brasileiros foram organizados para atendimento aos usuários dos serviços de saúde, mobilizando estruturas de triagem ambulatorial e internação de média e alta complexidade. Nas situações de impossibilidade do fluxo acontecer em uma só unidade de saúde, o transporte inter-hospitalar foi utilizado como estratégia complementar, conectando os pontos / unidades da RAS (Macedo et al, 2020). Cabe ressaltar que, além da organização das RAS e do processo de regulação, grande parte da responsabilidade pelo 
sucesso da assistência pré-hospitalar depende da iniciativa e da tomada de decisão dos profissionais, que devem ser baseadas no pensamento crítico e em evidências científicas (Eduardo et al, 2015; Motta et al, 2016).

Desde o início da pandemia de COVID-19 no Brasil, os profissionais de enfermagem têm desempenhado papel fundamental em todos os níveis de assistência, atuando no processo diagnóstico da doença, nos centros de testagem, na prevenção, no processo de imunização, no tratamento e na reabilitação dos pacientes acometidos . O aumento da demanda assistencial durante a pandemia de COVID-19 impôs novos desafios para as equipes de enfermagem, exigindo reorganização dos processos de trabalho e de tomada de decisão, além de fomentar desenvolvimento do raciocínio clínico, e a reflexão da prática assistencial (Antunes et al, 2021; Barros et al, 2020).

Entretanto, mediante às dificuldades impostas por este novo cenário, muitas reportagens apontaram impasses e questões relacionadas à assistência em saúde em todo país. No mês de janeiro de 2021, foram veiculadas nas mídias televisivas, de rádio e internet matérias abordando o caso de uma enfermeira que decidiu retirar de uma ambulância um paciente acometido pela COVID-19 e realizar o transporte de cerca de dois quilômetros empurrando uma maca em estrada de barro, enladeirada, com buracos (Conselho Federal de Enfermagem [COFEN], 2021). No momento do transporte o tráfego na estrada estava obstruído por fila de caminhões, impedindo que a ambulância prosseguisse e alcançasse seu destino. Além de gerar grande comoção entre a população brasileira, a situação em questão provocou discussão sobre os efeitos do excesso de pressão imposta pela pandemia de COVID-19 à rede de atenção à saúde, às condições estruturais do trabalho e, especialmente, ao processo de tomada de decisão pelos profissionais de saúde.

Este estudo contribui para a discussão deste contexto e sobre o impacto da tomada de decisão por profissionais de saúde que vêm atuando sob estado de tensão psicológica, em condições de trabalho desfavoráveis. Desta maneira, o objetivo do estudo foi descrever e analisar os fatores contribuintes e os riscos associados neste caso de tomada de decisão por uma enfermeira durante transporte inter-hospitalar de paciente acometido por COVID-19, amplamente divulgado nos meios de comunicação brasileiros.

\section{Metodologia}

Este é um estudo descritivo, teórico e reflexivo, cujo relato favorece o desenvolvimento do conhecimento por promover a organização e a descrição dos fatos, baseada em instrumentos de análise validados na literatura científica (Pereira et al, 2018; Rodrigues et al, 2020; Souza et al, 2020).

A fim de descrever e analisar a situação de interesse, o estudo foi organizado em três etapas, a saber: a) seleção das fontes de dados; b) construção do diagrama de causa e efeito; e c) construção da matriz de avaliação de riscos.

Foi realizada uma busca livre na internet utilizando os termos "enfermeira AND "estrada" AND "COVID-19", buscando-se identificar e selecionar materiais apresentando diferentes formas de abordagem e dados complementares sobre a situação, permitindo aos autores a possibilidade de uma análise aprofundada. Cada autor do estudo assistiu e realizou análise individual do material selecionado. Posteriormente, foi realizado um painel de discussões para a organização dos fatores contribuintes e os riscos associados à situação de interesse.

Para a identificação e análise dos fatores contribuintes foi elaborado o diagrama de causa e efeito, que é uma das ferramentas mais utilizadas para o gerenciamento de processos de qualidade. Esta técnica consiste em construir um diagrama para propiciar melhor análise sobre as possíveis causas de um determinado problema (Ishikawa \& Loftus, 1990; Liliana, 2016). Ademais, para categorizar e ranquear os riscos identificados, foi elaborada uma matriz de riscos, estratégia que consiste em construir uma tabela onde são representadas as probabilidades de ocorrência e as consequências (impactos) das possíveis situações de risco em um processo (Landell, 2016). 
O processo de coleta dos dados foi realizado durante os meses de fevereiro e março de 2021 e, a partir deste diagnóstico, foram realizadas análise e discussão do material obtido.

\section{Resultados}

Os resultados da análise da situação de interesse neste estudo, serão apresentados em três seções, de acordo com as etapas metodológicas.

-Seleção das fontes de dados.

O Quadro 1 apresenta a amostra selecionada como fonte de dados para o estudo, com uma seleção de doze vídeos e sete matérias escritas, com os respectivos hiperlinks para acesso.

Quadro 1 - Seleção de vídeos e matérias escritas para a coleta de dados - Rio de Janeiro, 2021.

\begin{tabular}{ll} 
Vídeos & Matérias escritas \\
\hline
\end{tabular}

https://www.youtube.com/watch?v=9nQ0JpC1qt4

https://www.youtube.com/watch?v=tm0Jf059sds

https://www.youtube.com/results?search_query=enfer meira+empurra+maca

https://www.youtube.com/watch?v=2t10k6ZsWBc

https://www.youtube.com/watch?v=a898d8OMKKo

https://www.youtube.com/watch?v=DlKaPuF0A70

https://www.youtube.com/watch?v=3bzG9CD6Ask http://www.cofen.gov.br/enfermeira-empurra-maca-com-paciente-por-2km-em-trecho-da-transamazonica-no-para_85537.html

https://recordtv.r7.com/balanco-geral/videos/enfermeira-empurra-macade-paciente-com-covid-19-por-quase-2-km-15022021

https://gmconline.com.br/noticias/geral/paciente-e-empurrado-porenfermeiro-em-maca-na-transamazonica-e-video-viraliza/

https://www.midiamax.com.br/brasil/2021/enfermeiro-empurra-maca-depaciente-com-covid-19-durante-2-km

https://noticias.uol.com.br/videos/2021/02/13/enfermeira-empurra-macacom-paciente-com-covid-19-na-rodovia-transamazonica.htm

https://amazonasatual.com.br/enfermeira-empurra-maca-em-trecho-deterra-da-transmazonica-apos-bloqueio-de-caminhoes/

https://brasil.estadao.com.br/noticias/geral,paciente-e-empurrado-porenfermeiro-em-maca-na-transamazonica-bloqueada-por-

carretas, 70003615197

https://www.youtube.com/watch?v=VXCUsUM1Whk

https://www.youtube.com/results?search_query=enfer

meira+empurra+maca

https://www.youtube.com/watch?v=2t10k6ZsWBc

https://www.youtube.com/watch?v=a898d8OMKKo

https://www.youtube.com/watch?v=DlKaPuF0A70

Fonte: Elaborado pelos autores. 


\section{- Construção do diagrama de causa e efeito}

O Diagrama de Causa e Efeito (DCE), também conhecido por Diagrama de Ishikawa, consiste em uma construção gráfica que tem como objetivo identificar a causa raiz de um determinado problema. Entre os componentes do DCE, destacamse o problema a ser avaliado (efeito), as categorias de fatores contribuintes e as causas específicas (Xu \& Dang, 2020).

Neste estudo, o problema a ser avaliado foi deinido como o transporte de maca na estrada, realizado por uma enfermeira. Para as categorias de fatores contribuintes, foi utilizada a classificação do Protocolo de Londres: paciente, tarefa/tecnologia, individuais, time (equipes), ambiente e institucional (Vincent, Taylor-Adams \& Stanhope, 1998).

O uso do Protocolo de Londres em uma investigação sistematizada, colabora para organizar as etapas, melhorar a qualidade da coleta de dados e auxiliar na reflexão de todas as dimensões dos Fatores Contribuintes, pontuando os aspectos mais importantes dos fatores humanos. As análises devem priorizar uma compreensão mais ampla da causa do incidente, com menos foco no indivíduo que comete um erro, e mais foco nos fatores organizacionais preexistentes que fornecem as condições e até induzem a ocorrência dos erros (Brasil, Agência Nacional de Vigilância Sanitária [ANVISA], 2017).

As causas independentes, organizadas de acordo com as categorias de fatores contribuintes são apresentadas na Figura 1.

Figura 1 - Diagrama de causa e efeito (Ishikawa) para a tomada de decisão da enfermeira - Rio de Janeiro, 2021.

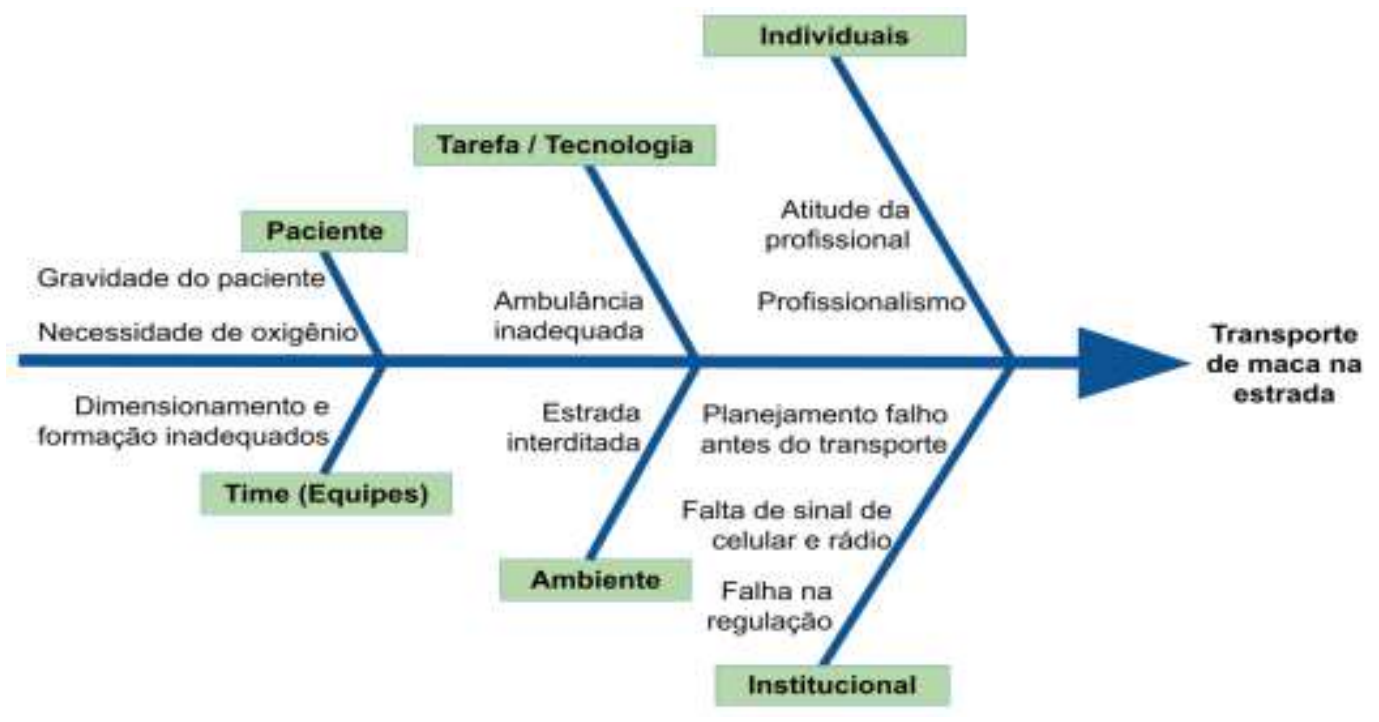

Fonte: Elaborado pelos autores.

Foram identificados as seguintes causas independentes para a tomada de decisão da profissional: Paciente: gravidade do paciente, necessidade de oxigenoterapia, Tarefa/Tecnologia: inadequações na ambulância, falha na comunicação por rádio e celular, Individual: atitude proativa da profissional, profissionalismo, Time/Equipes: dimensionamento e formação da equipe com inadequação, Ambiente: condições da estrada, Institucional: falha no planejamento antes do transporte, falha no processo de regulação.

Entre todas as causas relacionadas, destaca-se o fato de que a estrada estava interditada durante o momento do transporte, devido a uma manifestação de motoristas de caminhões. Apesar de este fator principal não depender dos profissionais de saúde ou dos serviços de regulação, outras questões periféricas foram cruciais para o processo de tomada de 
decisão.

\section{- Construção da matriz de avaliação de riscos}

A matriz de riscos é uma das ferramentas empregadas na análise de riscos de processos de várias naturezas que possibilita ao gestor visualizar todos os riscos que envolvem determinada decisão ou projeto e avaliar quais deles merecem maior atenção. Essa matriz reúne uma série de informações sobre potenciais riscos de forma objetiva e visualmente clara, o que facilita o entendimento pelas equipes envolvidas. $\mathrm{Na}$ matriz de riscos, são levados em consideração dois critérios: de probabilidade (chance de o risco identificado acontecer) e de impacto (nível de dano causado caso o risco se efetive), para determinar o nível de cada risco, ou seja, a classificação (Landell, 2016).

A matriz de riscos, com as informações sobre a profissional e sobre o paciente é apresentada no Quadro 2. Cada risco foi analisado independentemente por seis avaliadores, sendo os dados consolidados para resultar na configuração final.

Quadro 2 - Matriz de riscos para a profissional e para o paciente - Rio de Janeiro, 2021.

\begin{tabular}{|c|c|c|c|}
\hline Riscos & Probabilidade & $\begin{array}{l}\text { Consequência } \\
\text { (impacto) }\end{array}$ & $\begin{array}{l}\text { Classificação do } \\
\text { risco }\end{array}$ \\
\hline \multicolumn{4}{|c|}{ Para a profissional (Enfermeira) } \\
\hline Risco de queda devido às condições da estrada & $\frac{\text { Possível }(60 \%)}{\text { certo }(40 \%)}$ & $\frac{\text { Média }(80 \%)}{(20 \%)}$ / Extrema & Moderado \\
\hline Risco de lesões por queda & $\begin{array}{l}\text { Possível (40\%) / Quase } \\
\text { certo (40\%) / Rara (20\%) }\end{array}$ & $\frac{\text { Média }(80 \%)}{(20 \%)} /$ Extrema & Moderado \\
\hline $\begin{array}{l}\text { Risco de insuficiência de recursos para atendimento de } \\
\text { urgência na estrada }\end{array}$ & $\frac{\text { Quase certo (60\%) } /}{\text { Possivel }(40 \%)}$ & $\frac{\text { Extrema (80\%) / }}{\text { Desprezível (20\%) }}$ & $\underline{\text { Alto }}$ \\
\hline Risco de implicação ética em caso de danos ao paciente & $\begin{array}{l}\text { Possível }(60 \%) \text { / Quase } \\
\text { certo }(40 \%)\end{array}$ & $\begin{array}{c}\text { Média }(60 \%) / \text { Extrema } \\
(40 \%)\end{array}$ & Moderado \\
\hline \multicolumn{4}{|c|}{ Para o paciente } \\
\hline Risco de queda devido às condições da estrada & $\frac{\text { Possível }(60 \%)}{\text { certo }(40 \%)}$ & $\begin{array}{c}\text { Média }(40 \%) / \text { Extrema } \\
\qquad \underline{(60 \%)}\end{array}$ & Moderado \\
\hline Risco de lesões por queda & $\frac{\text { Possível }(60 \%)}{\text { certo }(40 \%)}$ & $\begin{array}{c}\text { Média }(40 \%) / \text { Extrema } \\
(60 \%)\end{array}$ & Moderado \\
\hline $\begin{array}{l}\text { Risco de morte por falta de oxigênio ou atendimento } \\
\text { inadequado na estrada }\end{array}$ & $\begin{array}{l}\text { Possível }(40 \%) / \text { Quase } \\
\text { certo }(60 \%)\end{array}$ & Extrema $(100 \%)$ & $\underline{\text { Alto }}$ \\
\hline Risco de descompensação hemodinâmica & $\frac{\text { Possível }(80 \%)}{\text { certo }(20 \%)}$ & $\frac{\text { Média }(60 \%)}{(40 \%)}$ / Extrema & Moderado \\
\hline $\begin{array}{l}\text { Risco de lesão por manipulação / disposição incorreta / } \\
\text { inadequada dos equipamentos }\end{array}$ & $\frac{\text { Possível }(80 \%) / \text { Quase }}{\text { certo }(20 \%)}$ & $\frac{\text { Média }(80 \%)}{(20 \%)}$ / Extrema & Moderado \\
\hline Risco de aspiração da poeira & $\begin{array}{c}\text { Possível }(40 \%) / \underline{\text { Rara }} \\
(60 \%)\end{array}$ & $\begin{array}{l}\text { Média }(60 \%) \text { / Extrema } \\
(20 \%) / \text { Desprezível }(20 \%)\end{array}$ & Moderado \\
\hline
\end{tabular}

Fonte: Elaborado pelos autores. 
De acordo com a avaliação dos especialistas, a maioria dos riscos foram classificados como moderados e apenas dois deles foram classificados como altos (probabilidade quase certa e consequência extrema): risco de insuficiência de recursos para atendimento de urgência na estrada - para a enfermeira e, risco de morte por falta de oxigênio. ou atendimento inadequado na estrada - para o paciente.

\section{Discussão}

A avaliação do contexto interno no sistema de gestão de riscos é essencial tanto no processo de organização quanto no suporte às tomadas de decisão (Nassar et al, 2020). Neste sentido, o presente estudo intenciona promover uma reflexão dos profissionais de saúde sobre os riscos e fatores contribuintes em tomadas de decisão crítica em qualquer cenário e no ambiente de transporte inter-hospitalar, visto que a Regulação da assistência deve estar pautada e pactuada com a avaliação, análise e gerenciamento dos riscos potenciais. Trata-se, na gestão de riscos, de conhecer as circunstâncias (perigos) que podem levar a um futuro dano e minimizar sua probabilidade de ocorrência (frequência) e consequência (gravidade) (Brasil, Agência Nacional de Vigilância Sanitária [ANVISA], 2017).

$\mathrm{O}$ sistema de regulação brasileiro tem sido questionado nos últimos anos devido, principalmente, à dificuldade de acesso, com isolamento e fragmentação dos serviços, precarização das políticas de pessoal e inadequação das estratégias de gestão (Cavalcanti, Cruz \& Padilha, 2018). Tais dificuldades podem ter sido responsáveis por alguns fatores contribuintes identificados neste estudo, como o planejamento inadequado do transporte do paciente e inadequação do tipo de veículo.

O transporte de pacientes críticos requer dimensionamento adequado de profissionais de saúde, com composição mínima de uma enfermeira e um médico, ambos com habilidades para atender situações de urgência e emergência (Brasil, Agência Nacional de Vigilância Sanitária [ANVISA], 2010; Brasil. Ministério da Saúde, 2002). Na situação analisada, apesar da gravidade do paciente, a profissional enfermeira possuía apenas o suporte do motorista da ambulância, o que contraria as necessidades da tarefa e acarreta sobrecarga física e emocional.

O risco de insuficiência de recursos para atendimento de urgência na estrada, caracterizado como alto, decorre tanto da possível ausência de equipamentos quanto da falta de profissionais para assistir o paciente no caso de uma instabilidade do paciente no transporte por maca. Situações de urgência, em geral, requerem materiais e instrumentos específicos e pessoal com qualificação e treinamento adequados (Oliveira et al, 2021).

Os principais riscos para o paciente foram o de morte por falta de oxigênio ou atendimento inadequado na estrada e o de descompensação hemodinâmica, ambos relacionados com as características clínicas das pessoas com COVID-19 e com o tempo de exposição ao risco durante todo o período de transporte. Segundo Córdova et al (2020), a dispnéia é um dos principais sintomas nesses pacientes, com maior prevalência entre o sétimo e o oitavo dia de internação e, com a aumento do tempo de transporte das duas horas inicialmente previstas para aproximadamente doze horas, houve grande probabilidade de o paciente apresentar repercussões graves relacionados a este sintoma.

Observa-se na situação em questão o impacto de fatores políticos e estruturais nos estados e municípios, que inicialmente não teriam correlação com o atendimento em saúde, mas que se tornam cruciais na determinação de desfechos, como a condição da estrada e sua obstrução por caminhões. Aliam-se a isso, as grandes distâncias que precisam ser percorridas no transporte inter-hospitalar nesta região e a carência de novas tecnologias de transporte para atendimento em saúde, como uso de helicópteros, que já são rotina nos grandes centros tanto para acesso à áreas remotas, como para otimização de tempo de atendimento, o que impacta positivamente nos resultados.

Em muitos momentos, a tomada de decisão constitui instrumento gerencial do processo de trabalho das enfermeiras. Esse processo decisório envolve a formação acadêmica, as experiências passadas, valores, crenças, filosofias, conhecimento 
técnico e habilidades, com foco na promoção do bem para o paciente. Desta maneira, esse arcabouço está fortemente marcado nas enfermeiras, na sua forma de pensar, saber e fazer sua assistência e de garantir a continuidade do cuidado, pautada no valor da vida (Rego \& Siqueira-Batista, 2009).

Segundo o código de ética dos profissionais de enfermagem, a profissão deve ser exercida com justiça, compromisso, eqüidade, resolutividade, dignidade, competência, responsabilidade, honestidade e lealdade (Conselho Federal de Enfermagem [COFEN], 2017). Tais argumentos justificam a tomada de decisão da enfermeira que, a partir da análise da situação e ponderação sobre os riscos e benefícios, tomou a decisão que julgou mais adequada diante da situação de crise.

Diante de todos esses pontos descritos, que tornam a análise desta situação bastante complicada, a possibilidade proposta neste estudo é de abordar essa discussão sob o ponto de vista do conceito de complexidade, com a intenção de fomentar novos debates. Nos sistemas complexos o comportamento não pode ser inferido a partir dos seus componentes somente, uma vez que os diferentes elementos interagem e produzem saídas que são imprevisíveis e não lineares, comportamentos esses que podem explicar diversos fenômenos na natureza humana e geral (Andrus, 2005; Lukosevicius, Marchisotti \& Soares, 2017).

A complexidade é um fenômeno quantitativo devido variedade de interações e interferências entre um número muito grande de unidades. Assim, ela compreende incertezas, indeterminações e fenômenos aleatórios, isto é, relaciona-se com a ideia de acaso (Morin, 2015). Desta maneira não cabem análises soltas ou excludentes de um ponto ou a fragmentação da situação, a partir dos diagnósticos feitos com as ferramentas de análise. Portanto, a tomada de decisão da enfermeira foi a única possível, quando entendemos o sistema interligado na rede dos vários problemas que encaminharam a tal desfecho.

A Figura 2 representa o modelo explicativo de Andrus (2005), para o pensamento adaptativo complexo, aplicado ao processo de tomada de decisão da enfermeira durante o transporte em questão neste estudo. À esquerda da figura, são observados todos os pontos interligados e todas as conexões consideradas para que o transporte inter-hospitalar aconteça e quando, durante o procedimento, a enfermeira de depara com uma situação emergente e inesperada (acaso), precisa decidir (pensamento adaptativo) que estratégia usar para tentar solucionar a situação imposta.

Diante da mudança no ambiente externo, caracterizada pela pandemia de COVID-19, e sofrendo a influência de todos os fatores relacionados com o transporte intra-hospitalar, as informações circulam no sistema sendo modificadas e voltando a influenciar no comportamento do mesmo seja como uma influência positiva (amplificada) ou negativa (amortecida). Entre os principais fatores de feedback positivo foram relacionados o pensamento crítico e a tomada de decisão da enfermeira durante a situação de crise, sua conduta ética e sentimento de satisfação profissional. Alguns fatores de feedback negativos que pressionaram a enfermeira durante a situação foram estresse físico e mental, o isolamento social, o sentimento de impotência e a culpa. 
Figura 2 - Comportamento adaptativo complexo aplicado à situação de tomada de decisão pela enfermeira - Rio de Janeiro, 2021.

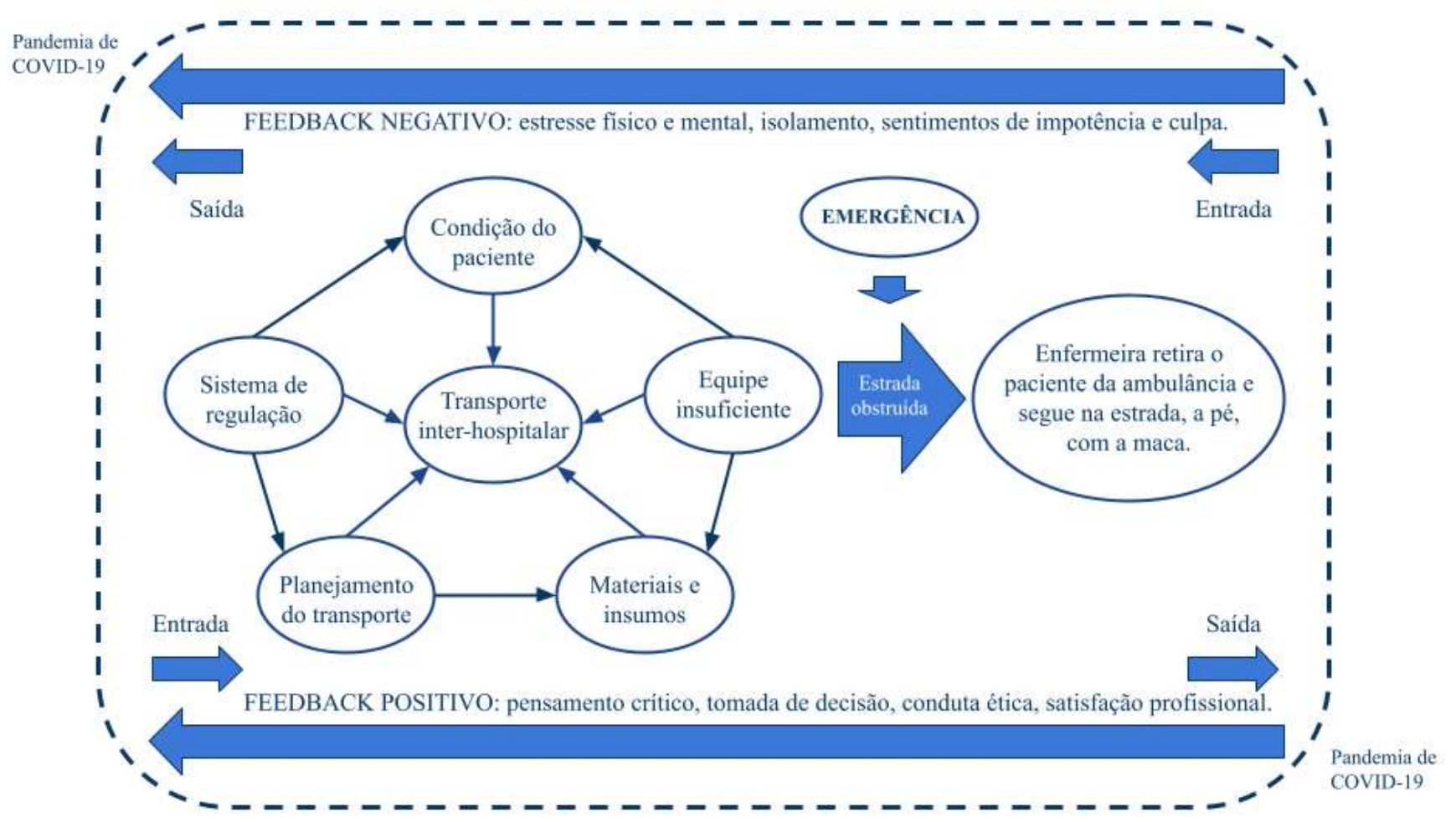

Fonte: Adaptado de Andrus (2005); Lukosevicius, Marchisotti \& Soares (2017).

Toda a situação vivida só pode ser discutida e compreendida quando percebe-se que: ainda há muito que se discutir quanto às condições de trabalho dos profissionais de saúde no país; que há uma grande necessidade de rever, implementar, e fiscalizar as políticas públicas de atenção à saúde principalmente quanto ao acesso aos serviços e aos conceitos de qualidade e segurança; que aspectos como economia, transporte, emprego, seguridade social também fazem parte do tema "saúde" e impactam de maneira decisiva na assistência prestada; e finalmente, que é preciso avançar para que situações parecidas e largamente noticiadas, como o caso em questão, não voltem a acontecer. A despeito de toda capacidade da profissional durante a tomada de decisão, não cabem aplausos e sim melhores condições para todos, profissionais e usuários.

\section{Conclusão}

Foi possível identificar os fatores contribuintes que guiaram a enfermeira no processo de tomada de decisão diante de uma situação crítica no atendimento pré-hospitalar. A análise dos riscos aos quais a enfermeira e o paciente ficaram expostos permitiu reflexão sobre a importância de um suporte adequado aos profissionais durante o atendimento pré-hospitalar, de maneira a minimizá-los e garantir a segurança para os pacientes e para as equipes.

É fundamental que os gestores públicos e os profissionais de saúde reflitam sobre a situação em destaque neste estudo e tracem estratégias para mitigar os riscos em situações semelhantes que possam ocorrer no futuro. A tomada de decisão sobre levar a maca pela estrada sofreu influência de uma série de fatores externos. Entretanto, pontua-se o profissionalismo, a dedicação e ética da profissional enfermeira que, diante de condições extremamente adversas, foi capaz de realizar a avaliação adequada e decidir pela melhor estratégia, conseguindo concluir o transporte sem qualquer dano para si mesma ou para o paciente. 
O presente estudo contribui para prática assistencial ao apresentar elementos com potencial para fomentar o pensamento crítico e a tomada de decisão clínica em situações complexas. No âmbito do ensino, os resultados, as análises e as discussões apresentados neste estudo podem servir como apoio teórico e metodológico para o desenvolvimento de estratégias de aprendizagem e desenvolvimento do raciocínio clínico em estudantes de enfermagem. Ao combinar as estratégias de análise de causas e riscos de eventos com o delineamento do comportamento adaptativo em sistemas complexos, este estudo se configura como uma iniciativa precursora na área da enfermagem.

Recomenda-se fortemente o desenvolvimento de estudos futuros aplicando a sequência de análises aqui apresentadas em outras situações de tomadas de decisões por enfermeiras ou outros profissionais de saúde.

\section{Referências}

Andrus, D. C. (2005). The Wiki and the Blog: Toward a Complex Adaptive Intelligence Community. Studies in Intelligence, 49(3). https://ssrn.com/abstract=755904.

Antunes, C. M. T. B., Luciano, C. C., Bahia. J. C. \& Bastos, R. M. A. F. (2021). Relato de experiência dos atendimentos de enfermagem em triagem para o diagnóstico da COVID-19 em profissionais da saúde. Nursing, 24(277), 5785-5792. https://doi.org/10.36489/nursing.2021v24i277p5785-5792

Barbosa, D. V. S., Barbosa, N. B. \& Najberg, E. (2016). Regulação em Saúde: desafios à governança do SUS. Cad. saúde colet, 24(1). https://doi.org/10.1590/1414-462X201600010106

Barros, A. L. B. L., Silva, V. M., Santana, R. F. S., Cavalcante, A. M. R. Z., Vitor, A. F., Lucena, A. F., Napoleão, A. A., Lopes, C. T., Primo, A. C., Carmona, E. V., Duran, E. C. M., Butcher, H. K., Lopes, J. L., Diaz, L. J. R., Cubas, M. R., Brandão, M. A. G., Lopes, M. V. O., Nóbrega, M. M. L., Almeida, M. A., Souza, P. A., Butcher, R. C. G. S., Jensen, R., Silva, R. S., Morais, S. C. R. V., Chianca, T. C. M. \& Santos, V. B. (2020). Brazilian Nursing Process Research Network contributions for assistance in the COVID-19 pandemic. Revista Brasileira de Enfermagem, 73( 2), 1-12. https://doi.org/10.1590/00347167-2020-0798.

Brasil. Ministério da Saúde (2002).Portaria no 2048. https://bvsms.saude.gov.br/bvs/saudelegis/gm/2002/prt2048_05_11_2002.html

Brasil. Ministério da Saúde (2010). Estabelece diretrizes para a organização da rede de atenção à saúde no âmbito do sistema único de saúde (SUS). https://bvsms.saude.gov.br/bvs/saudelegis/gm/2010/prt4279_30_12_2010.html

Brasil. Agência Nacional de Vigilância Sanitária (2010). Dispõe sobre os requisitos mínimos para funcionamento de Unidades de Terapia Intensiva e dá outras providências. https://bvsms.saude.gov.br/bvs/saudelegis/anvisa/2010/res0007_24_02_2010.html

Brasil. Ministério da Saúde (2011). Reformula a Política Nacional de Atenção às Urgências e institui a Rede de Atenção às Urgências no Sistema Único de Saúde (SUS). https://bvsms.saude.gov.br/bvs/saudelegis/gm/2011/prt1600_07_07_2011.html

Brasil. Agência Nacional de Vigilância Sanitária (2017). Gestão de Riscos e Investigação de Eventos Adversos Relacionados à Assistência à Saúde. https://segurancadopaciente.com.br/wp-content/uploads/2017/0

Cavalcanti, R. P., Cruz, D. F. \& Padilha, W. W. N. (2018). Desafios da regulação assistencial na organização do sistema único de saúde. Revista Brasileira De Ciências Da Saúde, 22(2), 181-188. https://doi.org/10.22478/ufpb.2317-6032.2018v22n2.31872

COFEN. Conselho Federal de Enfermagem. (2017). Código de ética dos profissionais de enfermagem. http://www.cofen.gov.br/resolucao-cofen-no5642017_59145.html

COFEN. Conselho Federal de Enfermagem. (2021). Enfermeira empurra maca com paciente por 2 km na Transamazônica, no Pará. http://www.cofen.gov.br/enfermeira-empurra-maca-com-paciente-por-2-km-em-trecho-da-transamazonica-no-para_85537.html

Córdova, L. D. S. (2021). Clinical characteristics of older patients with COVID-19: a systematic review of case reports. Dement Neuropsychol, 15 (1). https://doi.org/10.1590/1980-57642021dn15-010001

Eduardo, E. A., Peres, A. M., Almeida. M. L., Roglio. K. D. \& Bernardino, E. (2015). Analysis of the decision-making process of nurse managers: a collective reflection. Rev Bras Enferm, 68(4): 668-75. http://dx.doi.org/10.1590/0034-7167.2015680414i

Ishikawa, K. \& Loftus, J. H. (1990). Introduction to quality control, Ed. 3A Corporation.

Landell, H. (2016). The Risk Matrix as a tool for risk analysis - How to apply existing theories in practice in order to overcome its limitations. [Master dissertation, University of Gävle]. University of Gävle Repository. https://www.diva-portal.org/smash/get/diva2:944825/FULLTEXT01

Liliana, L. (2016). IOP Conf. Ser.: Mater. Sci. Eng., 161(012099). http://doi.org/10.1088/1757-899X/161/1/012099

Lukosevicius, A. P., Marchisotti, G. G. \& Soares, C. A. P. (2017). Panorama da complexidade: principais correntes, definições e constructos. Sistemas \&Amp; Gestão, 11(4), 455-465. https://doi.org/10.20985/1980-5160.2016.v11n4.1157

Macedo, T. R., Possoli, L., Rocha, D., Prado, S. S. \& Westphal, P. C. (2020). Regulação em saúde em tempos de Covid-19: um relato de experiência. Braz. J. Hea. Rev, 3(6), 19455-19465. https://doi.org/10.34119/bjhrv3n6-324 
Research, Society and Development, v. 10, n. 14, e237101421919, 2021

(CC BY 4.0) | ISSN 2525-3409 | DOI: http://dx.doi.org/10.33448/rsd-v10i14.21919

Morin, E. (2015). Introdução ao pensamento complexo, Ed. Sulina.

Motta, C. S., Oliveira, L.N., Silva, E. \& Batista, R. S. (2016). Tomada de decisão em (bio)ética clínica: abordagens contemporâneas. Revista Bioética, 24(2), 304-314. https://www.redalyc.org/comocitar.oa?id=361546419014

Nassar, P. R. B., Moraes, E. B., Braga, A. L. S., Souza, D. F., Christóvam, B. P. \& Neto, M. (2020) Gestão de risco no cenário COVID-19. Rev enferm UERJ, 28(55415). http://dx.doi.org/10.12957/reuerj.2020.55415

Oliveira, P. S., Diefenbach, G. D. F., Colomé, J., Buriol, D.; da Rosa, P. H. \& Ilha, S. (2021). Professional performance in urgencies/emergencies on basic units of health. Revista De Pesquisa Cuidado é Fundamental Online, 12, 820-826. https://doi.org/10.9789/2175-5361.rpcfo.v12.7556

Pereira, A.S., Shitsuka, D. M., Prreira, F. J. \& Shitsuka, R. (2018). Metodologia da pesquisa científica. UAB/NTE/UFSM

https://repositorio.ufsm.br/bitstream/handle/1/15824/Lic_Computacao_Metodologia-Pesquisa-Cientifica.pdf?sequence=1

Rego, S., Palácios, M. \& Siqueira-Batista, R. (2009). Bioética e a tomada de decisões: entre a clínica e a saúde pública. In: Bioética para profissionais da saúde, Ed. FIOCRUZ. https://doi.org/10.7476/9788575413906.0004.

Rodrigues, M. E. N. G., Belarmino, A.C., Custódio. L. L., Gomes, I. L. V. \& Júnior, A. R. F. F. (2020). Communication in health work during the COVID-19 pandemic. Invest. Educ. Enferm. 38(3). DOI: https://doi.org/10.17533/udea.iee.v38n3e09.

Souza, B. V. N., Teles, J. F. \& Oliveira, E. F. (2020). Dificultades y particularidades en el trabajo de los profesionales de atención prehospitalaria móvil: una revisión integradora. Enfermería Actual de Costa Rica, 38. http://dx.doi.org/10.15517/revenf.v0i38.36082

Souza, C. J. de, Silvino, Z. R., Joaquim, F. L., Souza, D. F. de, Christovam, B. P., Izu, M. \& Ferreira, A. de O. M. (2020). Academic maturity: a question of paradigma shift. Research, Society and Development, 9(6). https://doi.org/10.33448/rsd-v9i6.3437

Vincent, C., Taylor-Adams, S. \& Stanhope, N. (1998). Framework for analysing risk and safety in clinical medicine. BMJ, 11(316), 1154-1157. doi: 10.1136/bmj.316.7138.1154

Xu, Z. \& Dang, Y. (2020). Automated digital cause-and-effect diagrams to assist causal analysis in problem-solving: a data-driven approach. International Journal of Production Research, 58(17), 5359-5379. https://doi.org/10.1080/00207543.2020.1727043 